\title{
Insulin Increases Ceramide Synthesis in Skeletal Muscle
}

\author{
M. E. Hansen, ${ }^{1}$ T. S. Tippetts, ${ }^{1}$ M. C. Anderson, ${ }^{1}$ Z. E. Holub, ${ }^{1}$ E. R. Moulton, ${ }^{1}$ \\ A. C. Swensen, ${ }^{2}$ J. T. Prince, ${ }^{2}$ and B. T. Bikman ${ }^{1}$ \\ ${ }^{1}$ Department of Physiology and Developmental Biology, 593 WIDB, Brigham Young University, Provo, UT 84602, USA \\ ${ }^{2}$ Department of Chemistry and Biochemistry, Brigham Young University, Provo, UT 84602, USA
}

Correspondence should be addressed to B. T. Bikman; benjamin_bikman@byu.edu

Received 18 March 2014; Revised 15 April 2014; Accepted 22 April 2014; Published 18 May 2014

Academic Editor: Konstantinos Papatheodorou

Copyright ( 2014 M. E. Hansen et al. This is an open access article distributed under the Creative Commons Attribution License, which permits unrestricted use, distribution, and reproduction in any medium, provided the original work is properly cited.

\begin{abstract}
Aims. The purpose of this study was to determine the effect of insulin on ceramide metabolism in skeletal muscle. Methods. Skeletal muscle cells were treated with insulin with or without palmitate for various time periods. Lipids (ceramides and TAG) were isolated and gene expression of multiple biosynthetic enzymes were quantified. Additionally, adult male mice received daily insulin injections for 14 days, followed by muscle ceramide analysis. Results. In muscle cells, insulin elicited an increase in ceramides comparable to palmitate alone. This is likely partly due to an insulin-induced increase in expression of multiple enzymes, particularly SPT2, which, when knocked down, prevented the increase in ceramides. In mice, 14 days of insulin injection resulted in increased soleus ceramides, but not TAG. However, insulin injections did significantly increase hepatic TAG compared with vehicleinjected animals. Conclusions. This study suggests that insulin elicits an anabolic effect on sphingolipid metabolism in skeletal muscle, resulting in increased ceramide accumulation. These findings reveal a potential mechanism of the deleterious consequences of the hyperinsulinemia that accompanies insulin resistance and suggest a possible novel therapeutic target to mitigate its effects.
\end{abstract}

\section{Introduction}

Diabetes mellitus is diagnosed on the basis of elevated blood glucose, despite significant changes in other blood markers, such as lipids, lactate, or insulin [1,2]. The glucose emphasis is so strong that it provided the rationale for the disease name, which is understandable from a historic perspective, given that the main observable symptoms of the disease are indeed a consequence of glucose excess, including polyuria and its oft-noted sweetness [3]. However, while hyperglycemia represents the essential disorder of untreated type 1 diabetes mellitus, modern research reveals that many adverse consequences of type 2 diabetes mellitus are not a function of hyperglycemia, but rather of lipid and insulin excess $[1,4,5]$.

The initial challenge to the long-embraced glucosecentric view of diabetes was issued by Denis McGarry [4] and others since $[1,6]$, when he posited that hyperinsulinemia and hyperlipidemia may be the more likely pathophysiological mediators of type 2 diabetes. Despite being widely prescribed in type 2 diabetes, insulin administration correlates with higher mortality rates $[6,7]$, and hyperinsulinemia is a factor in multiple diseases, including hypertension [8], fatty-liver disease [9, 10], PCOS [11], Alzheimer's disease [12], and more [13-15]. Though the lipid-centric aspect of McGarry's paradigm focused on triacylglycerol and free fatty acids, the sphingolipid ceramide may be the more relevant lipid regarding the deleterious consequences of type 2 diabetes and/or hyperlipidemia [16]. Indeed, increased intracellular ceramide is associated with similar diseases as hyperinsulinemia $[10$, 17-19].

Ceramide is an established mediator of insulin resistance in response to multiple insults $[20,21]$ and in diverse tissues $[20,22]$. Additionally, muscle ceramide accumulation disrupts muscle metabolic function, altering mitochondrial structure, increasing ROS generation, and reducing mitochondrial respiration [23-25]. However, the role of insulin as an inducer of ceramide biosynthesis has never been explored. Not only would such a pathway identify a mechanism of negative feedback, wherein excessive insulin 
signaling downgrades its own signal, but also it would fit the general lipid-anabolic actions of insulin, even in skeletal muscle $[26,27]$. Thus, the purpose of these studies was to explore the novel role of insulin as an inducer of ceramide biosynthesis in skeletal muscle.

\section{Materials and Methods}

2.1. Animals. Sixteen-week-old male C57Bl/6 mice were separated into one of two groups at 12 weeks to receive daily saline or insulin $(0.75 \mathrm{U} / \mathrm{kg} / \mathrm{BW})$ injections for 14 days with free access to water and chow. $\mathrm{C} 57 \mathrm{Bl} / 6$ mice are commonly used as an ideal rodent model of exploring lifestyle risks associated with diabetes and obesity that mimic human responses [28]. Studies were conducted in accordance with the principles and procedures outlined in the National Institutes of Health Guide for the Care and Use of Laboratory Animals and were approved by the IACUC (Institutional Animal Care and Use Committee) at Brigham Young University.

2.2. Cell Culture. C2C12 murine myoblast cells were maintained in DMEM (Dulbecco's modified Eagle's medium) plus $10 \%$ FBS (Invitrogen). For differentiation into myotubes, $\mathrm{C} 2 \mathrm{C} 12$ myoblasts were grown to confluency and the medium was replaced with DMEM plus 10\% horse serum (Invitrogen). Myotubes were used for experiments on day 4 of differentiation. For fatty acid treatment, palmitic acid (SigmaAldrich, catalogue number P5585) was dissolved in EtOH (ethanol) and diluted to the desired concentration in DMEM containing 2\% ( $w / v)$ BSA (Sigma-Aldrich, A9576) and added to the cell culture at $0.5 \mathrm{mM}$, which is a physiological postprandial fatty acid concentration [29]. For sptlc2 (serine palmitoyltransferase, long chain base subunit 2, also known as SPT2) knockdown, procedures were followed according to the manufacturer's instructions (Santa Cruz Biotechnology, sc-77377).

2.3. Lipid Isolation and Analysis. For isolation of lipids, pellets were resuspended in $900 \mu \mathrm{L}$ of ice-cold chloroform/methanol (1:2), incubated for $15 \mathrm{~min}$ on ice, and then briefly vortex-mixed. Separation of aqueous and organic phases required addition of $400 \mu \mathrm{L}$ of ice-cold water and $300 \mu \mathrm{L}$ of ice-cold chloroform. The organic phase was collected into a fresh vial, and lipids were dried in a vacuum centrifuge (Eppendorf Concentrator Plus). Lipids were characterized and quantified using a shotgun lipidomics technique on a Thermo Scientific LTQ Orbitrap XL mass spectrometer. Evaporated lipid samples were resuspended in a $2: 1$ chloroform/methanol Folch solution $(200 \mu \mathrm{L})$. The resuspended lipids were then combined with a modified 2:1:1.25 chloroform/methanol/propan-2-ol Bligh and Dyer solution $(800 \mu \mathrm{L})$ with $15 \mathrm{mM}$ ammonium acetate acting as an ionizing adduct. A $1.74 \mu \mathrm{M}$ phosphatidylethanolamine internal standard $(1 \mu \mathrm{L})$ was spiked into each sample for mass calibration and characterization data alignment. Samples were analyzed using a $2.5 \mathrm{~min}$ mass-window-scanning method in positive-ion mode at a resolution of 60000 (fwhm (full width at half maximum) at $400 \mathrm{~m} / z$ ) for all primary
$\mathrm{MS}^{1}$ scans. $\mathrm{MS}^{2}$ (tandem MS) fragmentation data were also collected and manually verified for each mass window to give additional confidence to the correct identification of abundant lipid species. Three technical replicate mass spectrometer runs were performed on each sample. Samples were injected at $10 \mu \mathrm{L} / \mathrm{min}$ using a direct-inject ESI soft-ionization spray head from a Hamilton GASTIGHT glass syringe. The spray voltage and capillary temperature were maintained at $5.0 \mathrm{kV}$ and $275^{\circ} \mathrm{C}$, respectively. Each technical replicate was run in random in order to reduce systematic bias. Data were analyzed using in-house-developed peak summarization, recalibration, and lipid identification software using lipid database information from the Lipid MAPS (LIPID Metabolites and Pathways Strategy) Lipidomics Gateway database [30]. To ensure high-confidence identifications, an intensity threshold estimated to be $5 \%$ above the instrumental static signal was implemented. Lipid identities were only assigned when significantly observable peaks were identified in at least two of the three technical replicate runs. Nonzero lipid quantities were averaged from the replicate runs. The lipid species identified across different ionization states or with adducts were totaled together. Quantification was completed by normalizing total ion counts to the relative abundance of the internal standard that was spiked into each sample.

2.4. Protein Analysis. Tissue and cell extracts lysed and protein content was determined using a BCA protein assay (Pierce) and the sample volumes were adjusted so that precisely $50 \mu \mathrm{g}$ of protein was loaded into each lane. After the addition of sample buffer, samples were resolved by SDS/PAGE (10\% gel), transferred onto nitrocellulose membranes, and immunoblotted using methods described previously [31]. After incubation with primary antibody, blots were incubated with an HRP- (horseradish peroxidase-) conjugated secondary antibody. HRP activity was assessed with ECL solution (Thermo Scientific) and exposed to film. The antibodies used were anti-SPT2 (Abcam; ab23696) and antirabbit IgG (Cell Signaling Technology, 7074S).

2.5. Real-Time qPCR. Total RNA was extracted and purified from tissues using TRIzol (Invitrogen) according to the manufacturer's recommendations. cDNA was synthesized from mRNA via reverse transcription-PCR using a commercial cDNA synthesis kit with oligo(dT) primers (iScript Select cDNA Synthesis, Bio-Rad Laboratories). Quantitative real-time PCR was performed with Evagreen Ssofast (BioRad Laboratories) using a Bio-Rad Laboratories CFX Connect Real-Time PCR Detection system. Primer sequences were $5^{\prime}$-ACAGGATGCAGAAGGAGATTAC and $5^{\prime}$-CACAGAGTACTTGCGCTCAGGA as the forward and reverse primers, respectively, for $a c t b$ (actin), 5' -TACTCAGAGACCTCCAGCTG and $5^{\prime}$-CACCAGGGATATGCTGTCATC for sptlc1 (SPT1), 5'-GGAGATGCTGAAGCGGAAC and $5^{\prime}$-GTATGAGCTGCTGACAGGCA for sptlc2 (SPT2), $5^{\prime}$-CACCGGTACCTCGGAGCGGA and $5^{\prime}$-GTTTGGGATTGATGAACAGGGGT for des1 (Des1), $5^{\prime}$-CTGTTCTACTTGGCCTGTTG and $5^{\prime}$-TCATGCAGGAAGAACACGAG 
for lass1 (Cers1), 5'-CTCCAACGCTCACGAAATTC and $5^{\prime}$-ATGCAGACAGAAGATGAGTG for lass 5 (Cers5), $5^{\prime}$ GTTCGGAGCATTCAACGCTG and $5^{\prime}$-CTGAGTCGTGAAGACAGAGG for lass6 (Cers6), $5^{\prime}$-CTCGCTTGTCGTCTGCCT and $5^{\prime}$-TTGGCCCAGAACTCCTGTAG for FAS, and $5^{\prime}$-GTGCACAAGTGGTGCATCAG and $5^{\prime}$-CAGTGGGATCTGAGCCATCA for DGAT1. $\beta$-Actin reactions were performed side by side with every sample analyzed. Changes in the mRNA level of each gene for each treatment were normalized to that of the $\beta$-actin control mRNA according to Pfaffl [32].

\section{Results}

3.1. Insulin Increases Ceramide Biosynthesis in Muscle Cells. The primary observation of this report is that insulin treatment increases ceramides in murine myotubes. When treated with insulin $(50 \mathrm{nM})$ for $16 \mathrm{~h}$, ceramide levels increased roughly twofold (Figure 1). When assessing the insulin effects on gene expression, insulin increased the expression of genes encoding ceramide biosynthetic proteins SPT1 and 2, DES1 (Figure 2(a)), and FAS, involved in triacylglycerol synthesis (Figure 2(a)). Interestingly, the effects of insulin were comparable and even greater than palmitic acid (PA) treatment [20]. Moreover, the combined effects of both insulin and PA elicited an additive response with SPT2, resulting in a roughly $75 \%$ increase. In probing SPT2 gene expression further, we found that expression was significantly increased at $1 \mathrm{~h}$ of insulin treatment and peaked at $4 \mathrm{~h}$ (Figure 2(b)). A somewhat distinct trend was observed with actual ceramides, which peaked at $8 \mathrm{~h}$ (Figure 2(c)) and remained comparably elevated at $16 \mathrm{~h}$ of treatment.

3.2. SPT2 Is an Important Regulator of Insulin-Induced Ceramide Accrual. SPT2 was knocked down in murine myotubes via siRNA. To confirm the functional effects of the knockdown, SPT2 transcript level was measured in control (scramble) and SPT2 siRNA-treated cells with BSA and palmitic acid (PA) incubation, which is a well-established substrate and inducer of ceramide biosynthesis [33]. Transcript levels of SPT2 were reduced compared to control cells in both BSAand PA-treated conditions (Figure 3). Moreover, SPTKD cells failed to respond to PA treatment (Figure 3). Further, while ceramides levels were not significantly different in control and SPT2 KD cells in control conditions (CON), insulin (INS), PA, and insulin with PA (INS + PA) elicited a significant increase in ceramides in the control cells, but not the SPT2 KD cells (Figure 4(a)). Moreover, combination of insulin and PA (INS + PA) had a greater effect than INS and PA alone (Figure 4(a)). Interestingly, the SPT2 KD cells had higher TAG levels than control cells and experienced a significant increase in TAG levels with PA and INS + PA (Figure 4(b)).

3.3. Insulin Injections Increase Muscle Ceramides in Mice. Mice were injected with either insulin $(0.75 \mathrm{U} / \mathrm{kg})$ or PBS daily at the beginning of the light cycle for 14 days. Mice were allowed free access to food and water throughout

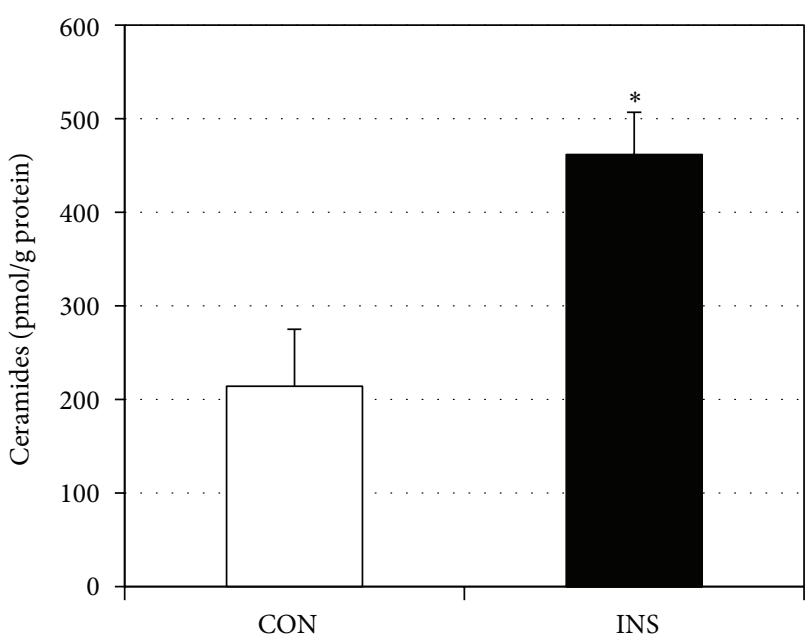

FIGURE 1: Murine myotubes were treated with vehicle (CON) or insulin (INS, $50 \mathrm{nM}$ ) for $16 \mathrm{~h}$. Following treatment, ceramides were isolated and quantified. ${ }^{*} P<0.05$.

the study period. At the conclusion of the study period, insulin-injected animals gained significantly greater body weight (Figure 5(a)), despite similar food consumption (Figure 5(b)). Gene expression levels in the muscle from insulin-injected animals tended to follow the trends observed in muscle cells. Specifically, levels of SPT1, SPT2, and FAS were elevated, but not DES1 and DGAT1 (Figure 6). Lastly, insulin injection had a particularly potent effect on muscle ceramide levels, but, as a comparison, not liver ceramides (Figure 7(a)). In contrast to ceramides, TAG levels in the soleus were not significantly elevated with insulin (Figure 7(b)). However, insulin injections did cause increased liver TAG (Figure 7(b)).

\section{Discussion}

This study evaluated the direct effect of insulin on skeletal muscle ceramide metabolism. The primary observation revealed that skeletal muscle cells respond to insulin treatments with a significant increase in ceramide, a finding further confirmed with increased ceramide in skeletal muscle from mice receiving daily insulin injections. This is, at least partly, a result of insulin-induced alterations in expression of enzymes involved in lipid handling (i.e., glycerolipid and sphingolipid). In particular, insulin increased expression of two isoforms of the initial and rate-limiting step in de novo ceramide biosynthesis (e.g., SPT1 and 2), as well as the final step, involving desaturation of the sphinganine backbone of dihydroceramide (DES1). Also, insulin increased FAS expression in both muscle cell cultures and whole muscle.

Our finding of increased ceramide accrual with insulin adds a layer of complexity to the abundant observations of increased skeletal muscle ceramide in insulin resistanthyperinsulinemic states [34-36]. Whereas the basic assumption is that ceramide exacerbates insulin resistance and this is well supported [33, 37-40], the present findings suggest that 


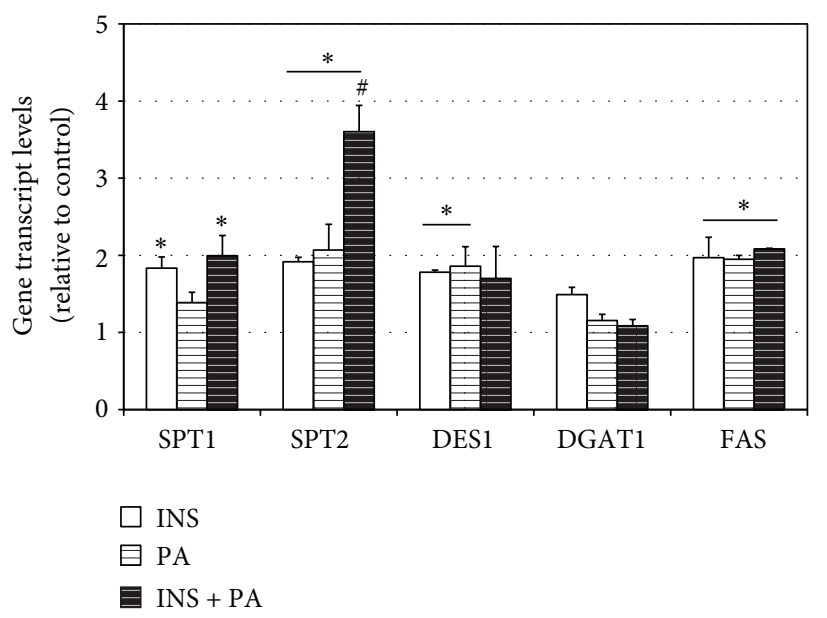

(a)

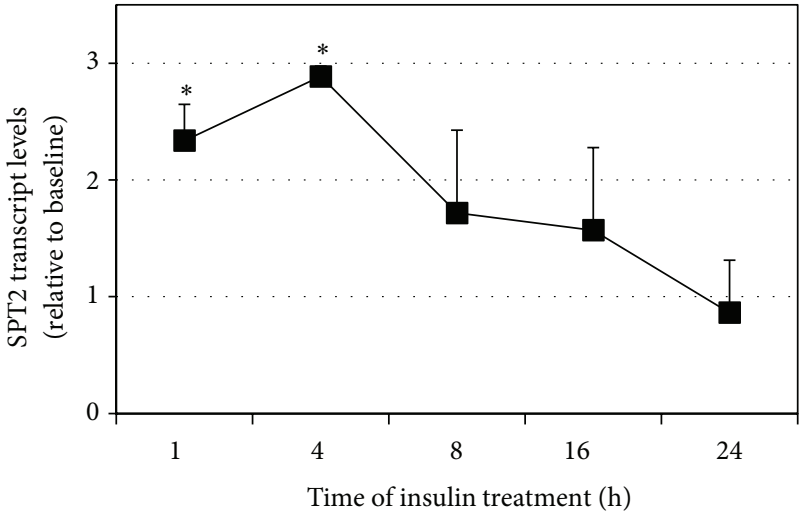

(b)

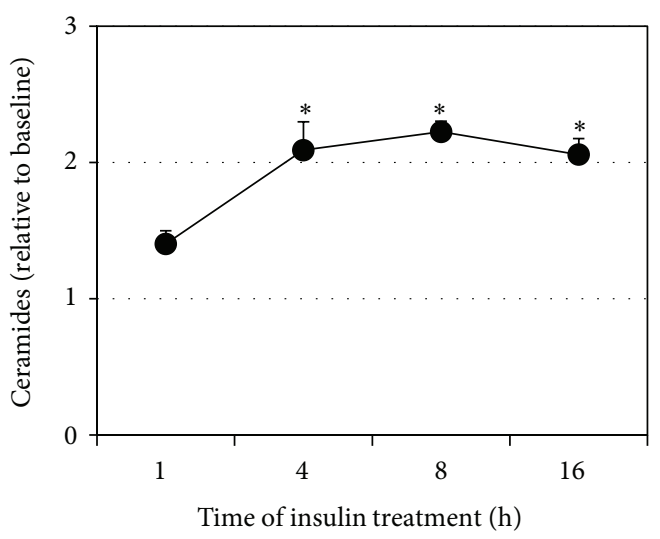

(c)

FIgURE 2: Murine myotubes were treated with insulin $(50 \mathrm{nM})$ with or without palmitic acid (PA, $0.5 \mathrm{mM})$ for $16 \mathrm{~h}$. (a) Following treatment, expressions of multiple enzymes involved with ceramide (SPT1, SPT2, and DES1) and TAG (DGAT1, FAS) metabolism were quantified. ${ }^{*} P<0.05$, treatment versus control. ${ }^{\#} P<0.05$, INS + PA versus other treatments. (b) The time course of insulin treatment (50 nM) revealed that SPT2 gene expression was significantly increased at $1 \mathrm{~h}$ and peaked at $4 \mathrm{~h} .{ }^{*} \mathrm{P}<0.05$, treatment versus control. (c) The time course of insulin treatment $(50 \mathrm{nM})$ revealed that ceramides were significantly increased at $4 \mathrm{~h}$ and sustained through $16 \mathrm{~h} .{ }^{*} P<0.05$, treatment versus control.

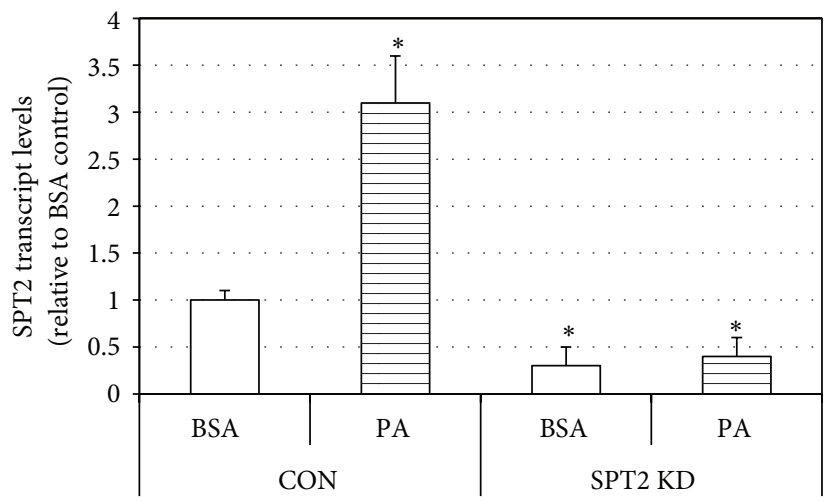

FiguRE 3: To confirm efficacy of SPT2 knockdown in murine myotubes, cells were treated with palmitic acid $(\mathrm{PA}, 0.5 \mathrm{mM}) .{ }^{*} P<0.05, \mathrm{PA}$ versus BSA. 


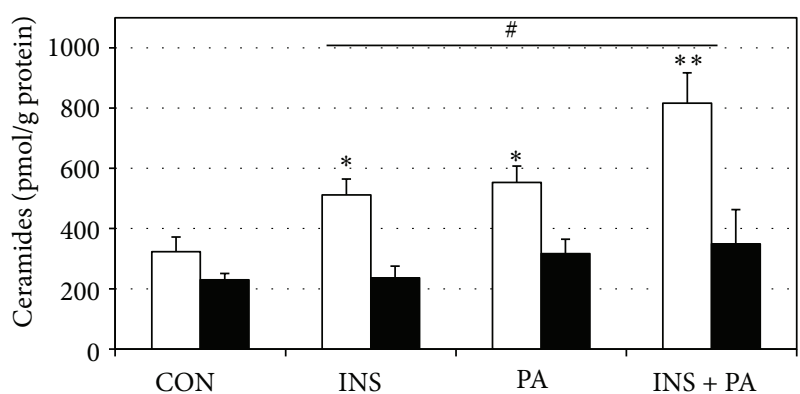

$\square \mathrm{CON}$

- SPT2 KD

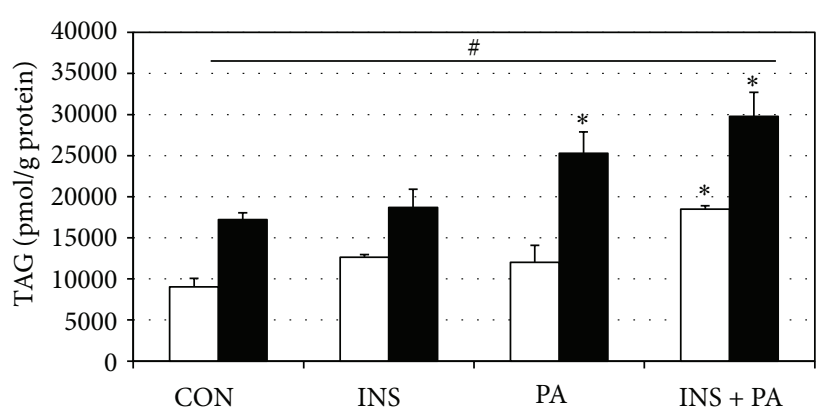

口 $\mathrm{CON}$

- SPT2 KD

(a)

(b)

FIGURE 4: Following SPT2 knockdown, myotubes were treated with insulin (INS, $50 \mathrm{nM}$ ), palmitic acid (PA, $0.5 \mathrm{mM}$ ), or both (INS + PA). (a) Ceramides were significantly comparably elevated with INS and PA treatments, but not with SPT2 KD $\left({ }^{*} P<0.05\right.$, treatment versus control). Both treatments together (INS + PA) resulted in an additive increase in ceramides $\left({ }^{* *} P<0.05\right.$, INS + PA versus INS and PA). SPT2 $\mathrm{KD}$ prevented treatment-induced increases in ceramide $\left({ }^{\#} P<0.05, \mathrm{CON}\right.$ (scramble) versus SPT2 KD). (b) TAG levels were elevated in PA and INS + PA treatments $\left({ }^{*} P<0.05\right.$, treatment versus control). SPT2 KD increased TAG in every condition $\left({ }^{\#} P<0.05\right.$, SPT2 versus CON (scramble)).

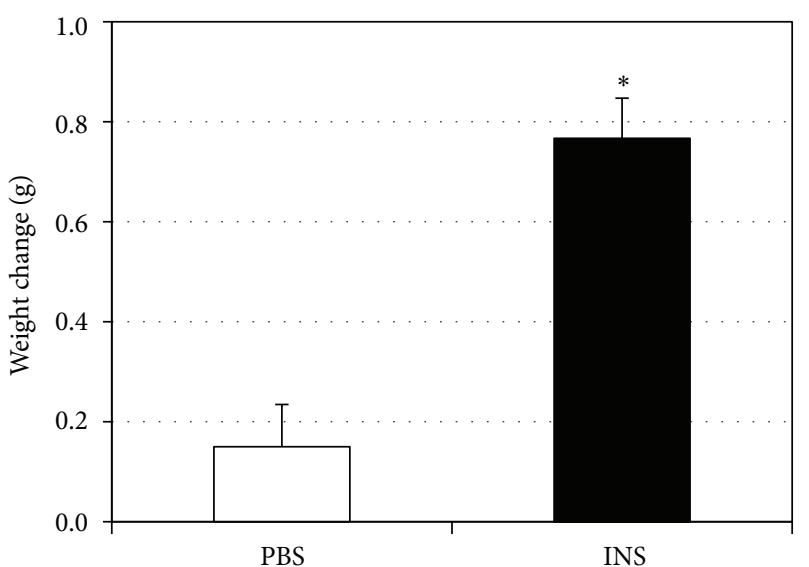

(a)

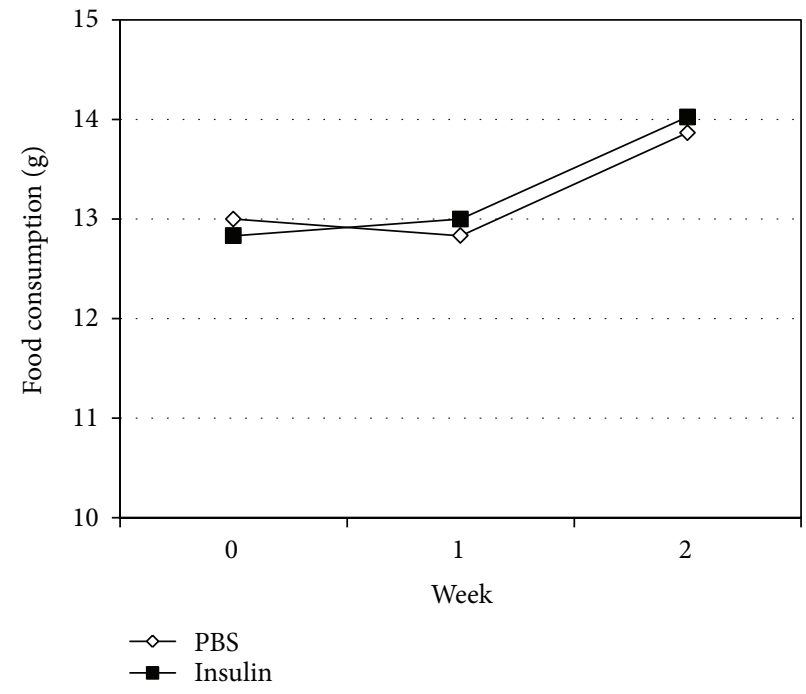

(b)

FIGURE 5: Adult male mice (12 weeks) were divided into two groups to receive insulin (INS, $50 \mathrm{nM}$ ) or vehicle (PBS) injections every morning for $14 \mathrm{~d}$. (a) Body weights were measured before and following treatment period. (b) Weekly food consumption was determined for the week prior to injection (week 0 ) and for each of the two following weeks of treatment. ${ }^{*} P<0.05$, INS versus PBS.

ceramide accumulation may be both a cause and consequence of hyperinsulinemia. Given ceramide's actions as an insulin antagonist, it is noteworthy that insulin increases ceramide accumulation in muscle. This phenomenon may indicate a degree of insulin self-regulation, wherein insulin downregulates its signaling when in excess, such as with the hyperinsulinemia accompanying insulin-resistant states. Interestingly, recent work by Zabielski et al. [41] established that insulin deprivation increased muscle ceramides by $\sim 50 \%$. Specifically, after inducing type 1 diabetes mellitus through streptozotocin treatment, Zabielski et al. [41] found that insulin deprivation increased quadriceps ceramide content. Thus, combined with our results, these reports collectively suggest that both lack of and excess of insulin increase muscle ceramide accrual.

Our findings of a significant increase in TAG with SPT2 KD are relevant given the expanding appreciation of TAG accrual in muscle being benign with regard to insulin resistance. Known as the athlete's paradox, this phenomenon was first observed by Goodpaster et al. [42]. Since that time, 


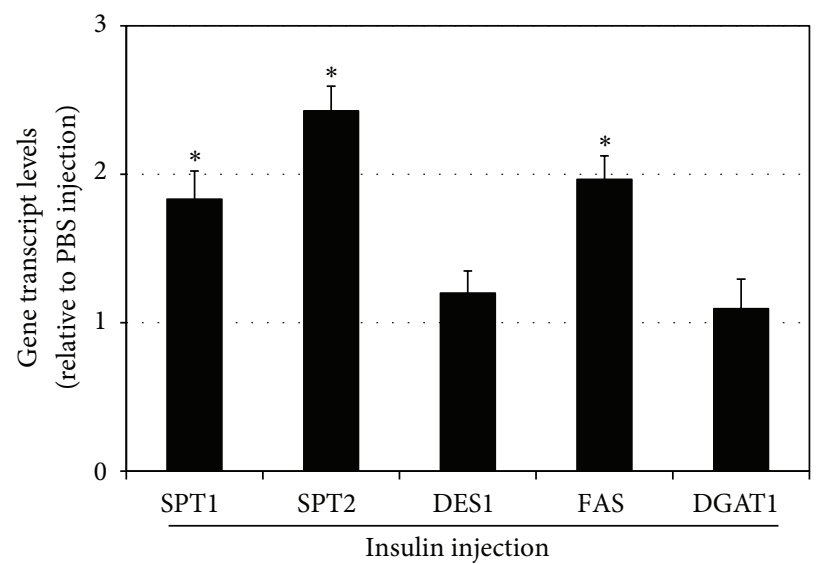

FIGURE 6: Adult male mice (12 weeks) were divided into two groups to receive insulin (INS, $50 \mathrm{nM}$ ) or vehicle (PBS) injections every morning for $14 \mathrm{~d}$. Soleus levels of genes of multiple enzymes involved with ceramide (SPT1, SPT2, and DES1) and TAG (DGAT1 and FAS) metabolism were quantified. ${ }^{*} P<0.05$, INS versus PBS.

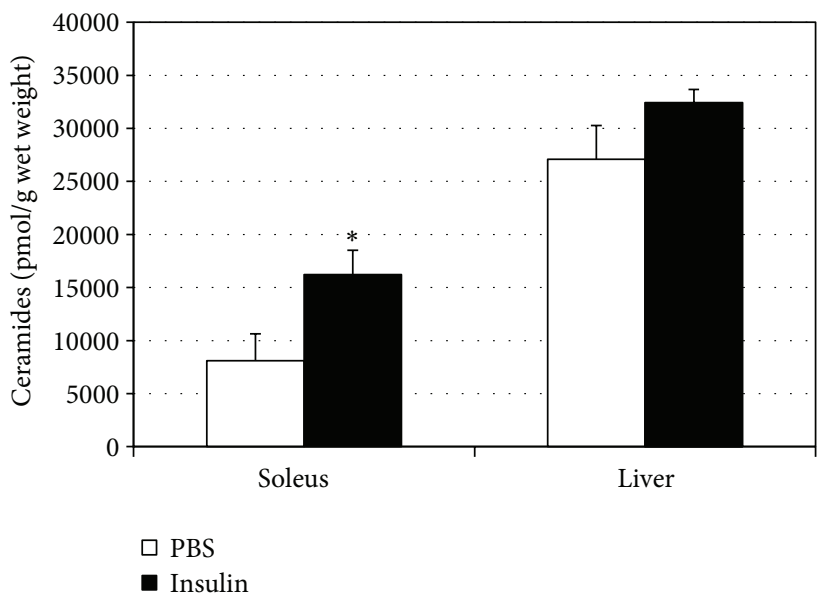

(a)

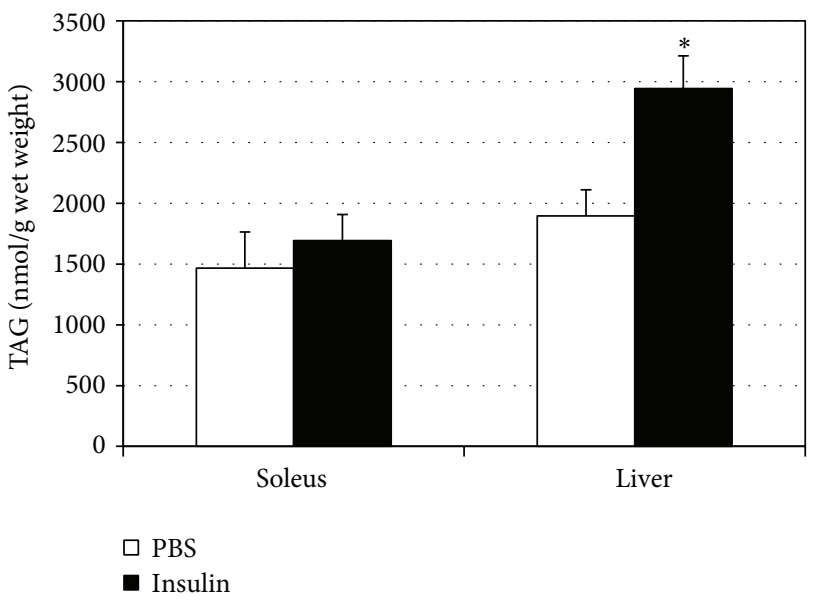

(b)

FIGURE 7: Following treatment period, soleus and liver were dissected from adult male mice receiving insulin (INS, $50 \mathrm{nM}$ ) or vehicle (PBS) injections every morning for $14 \mathrm{~d}$. Ceramides (a) and TAG (b) were quantified from both tissues. ${ }^{*} P<0.05$, insulin versus PBS.

shuttling lipid into the glycerolipid pathway in muscle is now considered a protective effect to insulin signaling. Liu et al. [43] found that mice that accumulate more TAG levels in muscle are protected against diet-induced insulin resistance. Importantly, this was associated with reduced ceramide content. We believe that a similar effect occurs with SPT2 KD; rather than shuttling carbons into sphingolipid biosynthesis with the various treatments (Figure 4), which is blunted due to knockdown of the rate-limiting step, the cell is increasing glycerolipid synthesis, evident by the increased TAG levels. Collectively, these observations suggest an insulin-sensitizing effect of ceramide inhibition and subsequent TAG synthesis.

Throughout these studies, palmitate was used as a positive control. Palmitate has long been known to increase ceramide accrual $[33,37]$, and it is noteworthy that insulin had a comparable effect on muscle ceramides as palmitate. Palmitate is important for ceramide biosynthesis for two reasons-as a substrate for ceramide creation (via SPT) and as an activator of ceramide biosynthetic pathways [20]. Our observation of an additive increase in ceramides with insulin and palmitate together in muscle cells is noteworthy because it reflects an environment that typifies insulin resistance-elevated insulin, increased circulating fatty acids, and increased muscle ceramides [34, 36, 44, 45]. The knowledge that both insulin and fatty acids increase ceramide highlights the potential importance of ceramide as a mediator of complications associated with elevations in both factors.

Multiple studies indicate that chronic exposure to insulin leads to insulin resistance [6], with dramatic increases in mortality from multiple diseases [46]. While the discussion is particularly relevant to type 2 diabetes, the ramifications similarly apply to type 1 diabetes. Deckert et al. [47] observed that type 1 diabetics that require less insulin have higher rates of survival. Altogether, these observations, in conjunction with 
our results, suggest the need for careful consideration when treating type 2 diabetes mellitus with insulin, especially when insulin levels are already elevated [6]. Whether ceramide is the primary regulator of reduced insulin sensitivity with chronic insulin is unknown.

While insulin resistance is known as a consequence of obesity [48], insulin is also critical in the expansion of adipose tissue that typifies obesity. Indeed, we observed a significant increase in body weight in the insulin-injected animals compared with the saline-injected mice (Figure 5(a)), despite no significant difference in food consumption. While the treatment period is relatively short, these findings nonetheless corroborate a substantial body of evidence showing the uniquely potent fattening effect of insulin [49-53], regardless of calories consumed [54, 55].

Insulin therapy is a common practice for treating type 2 diabetes mellitus. While this is undeniably effective at controlling blood glucose and mitigating the likelihood of hyperglycemia, the patient can suffer from the complications of hyperinsulinemia, including increased mortality [7] and weight gain [56]. Two thoughts arise from these observations. First, ideally the disease is detected in the early stage known as "prediabetes" or insulin resistance. However, this stage is typified by patients experiencing hyperinsulinemia, but not necessarily hyperglycemia. In other words, the pancreas is producing sufficient, if increased, insulin to maintain normoglycemia. Because blood glucose is typically used as the diagnostic marker of diabetes mellitus, but not insulin, many of these individuals remain undiagnosed [57]. Thus, insulin should be measured in clinical situations to allow the earlier detection of diabetes mellitus. Second, increasing efforts should be focused on lifestyle to control glucose and insulin, particularly diet. Carbohydrate-restricted diets, even in the absence of calorie restriction, are highly effective at reducing blood glucose and type 2 diabetics are often able to reduce the dose of or stop taking diabetic medications altogether [58].

\section{Conclusions}

In conclusion, our findings of increased ceramide biosynthesis with insulin provide a possible mechanism to partly explain the substantial evidence linking hyperinsulinemic conditions (e.g., insulin resistance and type 2 diabetes) to multiple disease states, especially vascular complications. For example, insulin resistance is associated with a significant increase in atherosclerosis [59] and ceramide increases atherosclerotic lesion development [60]. Similarly, insulin resistance is a common causal factor in hypertension [61], and vascular ceramide accrual compromises vasodilation $[62,63]$, increasing blood pressure. Thus, anticeramide therapies may prove to be a viable therapy for combating certain insulininduced disorders, which is the focus of ongoing efforts.

\section{Conflict of Interests}

The authors declare that there is no conflict of interests regarding the publication of this paper.

\section{References}

[1] W. J. Pories and G. L. Dohm, "Diabetes: have we got it all wrong? Hyperinsulinism as the culprit: surgery provides the evidence," Diabetes Care, vol. 35, no. 12, pp. 2438-2442, 2012.

[2] G. M. Reaven, C. Hollenbeck, C.-Y. Jeng, M. S. Wu, and Y.-D. I. Chen, "Measurement of plasma glucose, free fatty acid, lactate, and insulin for $24 \mathrm{~h}$ in patients with NIDDM," Diabetes, vol. 37, no. 8, pp. 1020-1024, 1988.

[3] A. K. Das and S. Shah, "History of diabetes: from ants to analogs," The Journal of the Association of Physicians of India, vol. 59, pp. 6-7, 2011.

[4] J. D. McGarry, "What if Minkowski had been ageusic? An alternative angle on diabetes," Science, vol. 258, no. 5083, pp. 766770, 1992.

[5] J. A. Chavez and S. A. Summers, "Lipid oversupply, selective insulin resistance, and lipotoxicity: molecular mechanisms," Biochimica et Biophysica Acta, vol. 1801, no. 3, pp. 252-265, 2010.

[6] M. H. Shanik, Y. Xu, J. Skrha, R. Dankner, Y. Zick, and J. Roth, "Insulin resistance and hyperinsulinemia: is hyperinsulinemia the cart or the horse?" Diabetes Care, vol. 31, supplement 2, pp. S262-S268, 2008.

[7] C. J. Currie, J. R. Peters, A. Tynan et al., "Survival as a function of $\mathrm{HbAlc}$ in people with type 2 diabetes: a retrospective cohort study," The Lancet, vol. 375, no. 9713, pp. 481-489, 2010.

[8] E. Ferrannini, G. Buzzigoli, R. Bonadonna et al., "Insulin resistance in essential hypertension," The New England Journal of Medicine, vol. 317, no. 6, pp. 350-357, 1987.

[9] K. M. Utzschneider and S. E. Kahn, "Review: the role of insulin resistance in nonalcoholic fatty liver disease," The Journal of Clinical Endocrinology and Metabolism, vol. 91, no. 12, pp. 47534761, 2006.

[10] B. T. Bikman and S. A. Summers, "Sphingolipids and hepatic steatosis," Advances in Experimental Medicine and Biology, vol. 721, pp. 87-97, 2011.

[11] A. Dunaif, "Insulin resistance and the polycystic ovary syndrome: mechanism and implications for pathogenesis," Endocrine Reviews, vol. 18, no. 6, pp. 774-800, 1997.

[12] G. S. Watson and S. Craft, "The role of insulin resistance in the pathogenesis of Alzheimer's disease: implications for treatment," CNS Drugs, vol. 17, no. 1, pp. 27-45, 2003.

[13] K. Murakami, Y. Shigematsu, M. Hamada, and J. Higaki, "In-sulin resistance in patients with hypertrophic cardiomyopthy," Circulation Journal, vol. 68, no. 7, pp. 650-655, 2004.

[14] C. Guillet and Y. Boirie, "Insulin resistance: a contributing factor to age-related muscle mass loss?" Diabetes \& Metabolism, vol. 31, no. 2, pp. S20-S26, 2005.

[15] R. A. DeFronzo and E. Ferrannini, "Insulin resistance: a multifaceted syndrome responsible for NIDDM, obesity, hypertension, dyslipidemia, and atherosclerotic cardiovascular disease," Diabetes Care, vol. 14, no. 3, pp. 173-194, 1991.

[16] J. M. Haus, S. R. Kashyap, T. Kasumov et al., "Plasma ceramides are elevated in obese subjects with type 2 diabetes and correlate with the severity of insulin resistance," Diabetes, vol. 58 , no. 2 , pp. 337-343, 2009.

[17] L. J. A. Spijkers, R. F. P. van den Akker, B. J. A. Janssen et al., "Hypertension is associated with marked alterations in sphingolipid biology: a potential role for ceramide," PLOS ONE, vol. 6, no. 7, Article ID e21817, 2011. 
[18] N. Marks, M. J. Berg, M. Saito, and M. Saito, "Glucosylceramide synthase decrease in frontal cortex of Alzheimer brain correlates with abnormal increase in endogenous ceramides: consequences to morphology and viability on enzyme suppression in cultured primary neurons," Brain Research, vol. 1191, pp. 136-147, 2008.

[19] R. G. Cutler, J. Kelly, K. Storie et al., "Involvement of oxidative stress-induced abnormalities in ceramide and cholesterol metabolism in brain aging and Alzheimer's disease," Proceedings of the National Academy of Sciences of the United States of America, vol. 101, no. 7, pp. 2070-2075, 2004.

[20] W. L. Holland, B. T. Bikman, L.-P. Wang et al., "Lipid-induced insulin resistance mediated by the proinflammatory receptor TLR4 requires saturated fatty acid-induced ceramide biosynthesis in mice," The Journal of Clinical Investigation, vol. 121, no. 5, pp. 1858-1870, 2011.

[21] W. L. Holland, J. T. Brozinick, L.-P. Wang et al., "Inhibition of ceramide synthesis ameliorates glucocorticoid-, saturated-fat-, and obesity-induced insulin resistance," Cell Metabolism, vol. 5, no. 3, pp. 167-179, 2007.

[22] J. W. Hill, C. F. Elias, M. Fukuda et al., "Direct insulin and leptin action on pro-opiomelanocortin neurons is required for normal glucose homeostasis and fertility," Cell Metabolism, vol. 11, no. 4, pp. 286-297, 2010.

[23] M. E. Smith, T. S. Tippetts, E. S. Brassfield et al., "Mitochondrial fission mediates ceramide-induced metabolic disruption in skeletal muscle," The Biochemical Journal, vol. 456, pp. 427-439, 2013.

[24] B. T. Bikman and S. A. Summers, "Ceramides as modulators of cellular and whole-body metabolism," The Journal of Clinical Investigation, vol. 121, no. 11, pp. 4222-4230, 2011.

[25] B. T. Bikman, "A role for sphingolipids in the pathophysiology of obesity-induced inflammation. Cellular and molecular life sciences," Cellular and Molecular Life Sciences, vol. 69, no. 13, pp. 2135-2146, 2012.

[26] S. R. Commerford, L. Peng, J. J. Dubé, and R. M. O’Doherty, "In vivo regulation of SREBP-1c in skeletal muscle: effects of nutritional status, glucose, insulin, and leptin," American Journal of Physiology: Regulatory Integrative and Comparative Physiology, vol. 287, no. 1, pp. R218-R227, 2004.

[27] I. Guillet-Deniau, V. Mieulet, S. L. Lay et al., "Sterol regulatory element binding protein-1c expression and action in rat muscles: Insulin-like effects on the control of glycolytic and lipogenic enzymes and UCP3 gene expression," Diabetes, vol. 51, no. 6, pp. 1722-1728, 2002.

[28] K. Srinivasan and P. Ramarao, "Animal models in type 2 diabetes research: an overview," The Indian Journal of Medical Research, vol. 125, no. 3, pp. 451-472, 2007.

[29] D. A. Fraser, J. Thoen, A. C. Rustan, Ø. Førre, and J. KjeldsenKragh, "Changes in plasma free fatty acid concentrations in rheumatoid arthritis patients during fasting and their effects upon T-lymphocyte proliferation," Rheumatology, vol. 38, no. 10, pp. 948-952, 1999.

[30] E. Fahy, S. Subramaniam, R. C. Murphy et al., "Update of the LIPID MAPS comprehensive classification system for lipids," Journal of Lipid Research, vol. 50, pp. S9-S14, 2009.

[31] K. A. Erickson, M. E. Smith, T. S. Anthonymuthu et al., "AICAR inhibits ceramide biosynthesis in skeletal muscle," Diabetology \& Metabolic Syndrome, vol. 4, no. 1, article 45, 2012.

[32] M. W. Pfaffl, "A new mathematical model for relative quantification in real-time RT-PCR," Nucleic Acids Research, vol. 29, no. 9, article e45, 2001.
[33] B. T. Bikman, Y. Guan, G. Shui et al., "Fenretinide prevents lipidinduced insulin resistance by blocking ceramide biosynthesis," The Journal of Biological Chemistry, vol. 287, no. 21, pp. 1742617437, 2012.

[34] J. M. Adams II, T. Pratipanawatr, R. Berria et al., "Ceramide content is increased in skeletal muscle from obese insulinresistant humans," Diabetes, vol. 53, no. 1, pp. 25-31, 2004.

[35] M. Straczkowski, I. Kowalska, M. Baranowski et al., "Increased skeletal muscle ceramide level in men at risk of developing type 2 diabetes," Diabetologia, vol. 50, no. 11, pp. 2366-2373, 2007.

[36] P. M. Coen, J. J. Dubé, F. Amati et al., "Insulin resistance is associated with higher intramyocellular triglycerides in type I but not type II myocytes concomitant with higher ceramide content," Diabetes, vol. 59, no. 1, pp. 80-88, 2010.

[37] J. A. Chavez, T. A. Knotts, L.-P. Wang et al., "A role for ceramide, but not diacylglycerol, in the antagonism of insulin signal transduction by saturated fatty acids," The Journal of Biological Chemistry, vol. 278, no. 12, pp. 10297-10303, 2003.

[38] C. Schmitz-Peiffer, D. L. Craig, and T. J. Biden, "Ceramide generation is sufficient to account for the inhibition of the insulin-stimulated PKB pathway in C2C12 skeletal muscle cells pretreated with palmitate," The Journal of Biological Chemistry, vol. 274, no. 34, pp. 24202-24210, 1999.

[39] F. Amati, J. J. Dubé, E. Alvarez-Carnero et al., "Skeletal muscle triglycerides, diacylglycerols, and ceramides in insulin resistance: another paradox in endurance-trained athletes?" Diabetes, vol. 60, no. 10, pp. 2588-2597, 2011.

[40] W. L. Holland, R. A. Miller, Z. V. Wang et al., "Receptormediated activation of ceramidase activity initiates the pleiotropic actions of adiponectin," Nature Medicine, vol. 17, no. 1, pp. 55-63, 2011.

[41] P. Zabielski, A. U. Blachnio-Zabielska, I. R. Lanza et al., "Impact of insulin deprivation and treatment on sphingolipid distribution in different muscle subcellular compartments of streptozotocin-diabetic C57Bl/6 mice," American Journal of Physiology Endocrinology and Metabolism, 2013.

[42] B. H. Goodpaster, J. He, S. Watkins, and D. E. Kelley, "Skeletal muscle lipid content and insulin resistance: evidence for a paradox in endurance-trained athletes," The Journal of Clinical Endocrinology and Metabolism, vol. 86, no. 12, pp. 5755-5761, 2001.

[43] L. Liu, Y. Zhang, N. Chen, X. Shi, B. Tsang, and Y.-H. Yu, "Upregulation of myocellular DGAT1 augments triglyceride synthesis in skeletal muscle and protects against fat-induced insulin resistance," The Journal of Clinical Investigation, vol. 117, no. 6, pp. 1679-1689, 2007.

[44] J. A. Lopez-Velazquez, L. D. Carrillo-Cordova, N. C. ChavezTapia, M. Uribe, and N. Mendez-Sanchez, "Nuclear receptors in nonalcoholic Fatty liver disease," Journal of Lipids, vol. 2012, Article ID 139875, 10 pages, 2012.

[45] S. Li, M. S. Brown, and J. L. Goldstein, "Bifurcation of insulin signaling pathway in rat liver: mTORC1 required for stimulation of lipogenesis, but not inhibition of gluconeogenesis," Proceedings of the National Academy of Sciences of the United States of America, vol. 107, no. 8, pp. 3441-3446, 2010.

[46] F. S. Facchini, N. Hua, F. Abbasi, and G. M. Reaven, "Insulin resistance as a predictor of age-related diseases," The Journal of Clinical Endocrinology and Metabolism, vol. 86, no. 8, pp. 35743578, 2001.

[47] T. Deckert, J. E. Poulsen, and M. Larsen, "Prognosis of diabetics with diabetes onset before the age of thirtyone. II. Factors 
influencing the prognosis," Diabetologia, vol. 14, no. 6, pp. 371$377,1978$.

[48] G. S. Hotamisligil, P. Peraldi, A. Budavari, R. Ellis, M. F. White, and B. M. Spiegelman, "IRS-1-mediated inhibition of insulin receptor tyrosine kinase activity in TNF-alpha- and obesityinduced insulin resistance," Science, vol. 271, no. 5249, pp. 665668, 1996.

[49] P. A. Velasquez-Mieyer, P. A. Cowan, K. L. Arheart et al., "Suppression of insulin secretion is associated with weight loss and altered macronutrient intake and preference in a subset of obese adults," International Journal of Obesity, vol. 27, no. 2, pp. 219-226, 2003.

[50] B. M. King, R. L. Smith, and L. A. Frohman, "Hyperinsulinemia in rats with ventromedial hypothalamic lesions: role of hyperphagia," Behavioral Neuroscience, vol. 98, no. 1, pp. 152-155, 1984.

[51] B. E. Hustvedt and A. Lovo, "Correlation between hyperinsulinemia and hyperphagia in rats with ventromedial hypothalamic lesions," Acta Physiologica Scandinavica, vol. 84, no. 1, pp. 29-33, 1972.

[52] M. G. Carlson and P. J. Campbell, "Intensive insulin therapy and weight gain in IDDM," Diabetes, vol. 42, no. 12, pp. 1700-1707, 1993.

[53] S. Mäkimattila, K. Nikkilä, and H. Yki-Järvinen, "Causes of weight gain during insulin therapy with and without metformin in patients with Type II diabetes mellitus," Diabetologia, vol. 42, no. 4, pp. 406-412, 1999.

[54] N. Torbay, E. F. Bracco, A. Geliebter, I. M. Stewart, and S. A. Hashim, "Insulin increases body fat despite control of food intake and physical activity," The American Journal of Physiology, vol. 248, no. 1, part 2, pp. R120-R124, 1985.

[55] J. E. Cox and T. L. Powley, "Development of obesity in diabetic mice pair fed with lean siblings," Journal of Comparative and Physiological Psychology, vol. 91, no. 2, pp. 347-358, 1977.

[56] D. M. Nathan, J. B. Buse, M. B. Davidson et al., "Medical management of hyperglycemia in type 2 diabetes: a consensus algorithm for the initiation and adjustment of therapy: a consensus statement of the American Diabetes Association and the European Association for the Study of Diabetes," Diabetes Care, vol. 32, no. 1, pp. 193-203, 2009.

[57] American Diabetes Association, "Diagnosis and classification of diabetes mellitus," Diabetes Care, vol. 33, supplement 1, pp. S62-S69, 2010.

[58] W. S. Yancy Jr., M. Foy, A. M. Chalecki, M. C. Vernon, and E. C. Westman, "A low-carbohydrate, ketogenic diet to treat type 2 diabetes," Nutrition \& Metabolism, vol. 2, article 34, 2005.

[59] R. W. Stout, "Insulin-stimulated lipogenesis in arterial tissue in relation to diabetes and atheroma," The Lancet, vol. 2, no. 7570, pp. 702-703, 1968.

[60] J. Bismuth, P. Lin, Q. Yao, and C. Chen, "Ceramide: a common pathway for atherosclerosis?” Atherosclerosis, vol. 196, no. 2, pp. 497-504, 2008.

[61] G. M. Reaven, H. Lithell, and L. Landsberg, "Hypertension and associated metabolic abnormalities-the role of insulin resistance and the sympathoadrenal system," The New England Journal of Medicine, vol. 334, no. 6, pp. 374-381, 1996.

[62] D. X. Zhang, A.-P. Zou, and P.-L. Li, "Ceramide reduces endothelium-dependent vasodilation by increasing superoxide production in small bovine coronary arteries," Circulation Research, vol. 88, no. 8, pp. 824-831, 2001.
[63] Q. J. Zhang, W. L. Holland, L. Wilson et al., "Ceramide mediates vascular dysfunction in diet-induced obesity by PP2Amediated dephosphorylation of the eNOS-Akt complex," Diabetes, vol. 61, no. 7, pp. 1848-1859, 2012. 


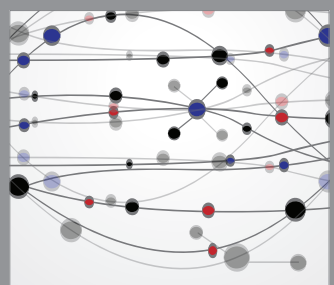

The Scientific World Journal
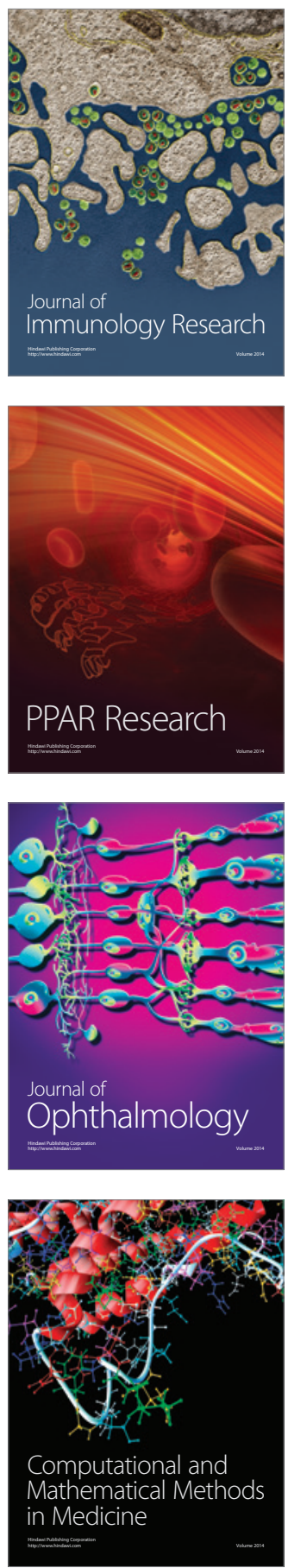

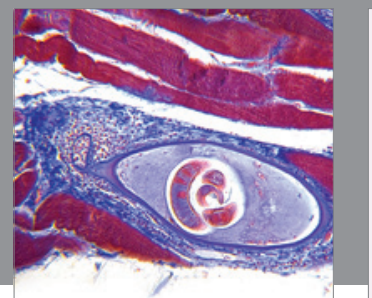

Gastroenterology

Research and Practice
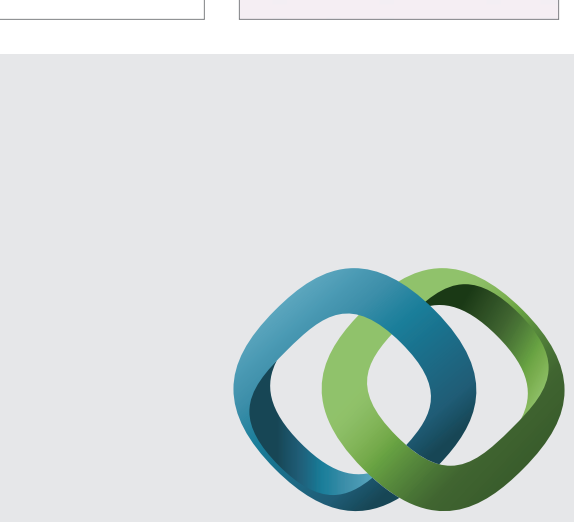

\section{Hindawi}

Submit your manuscripts at

http://www.hindawi.com
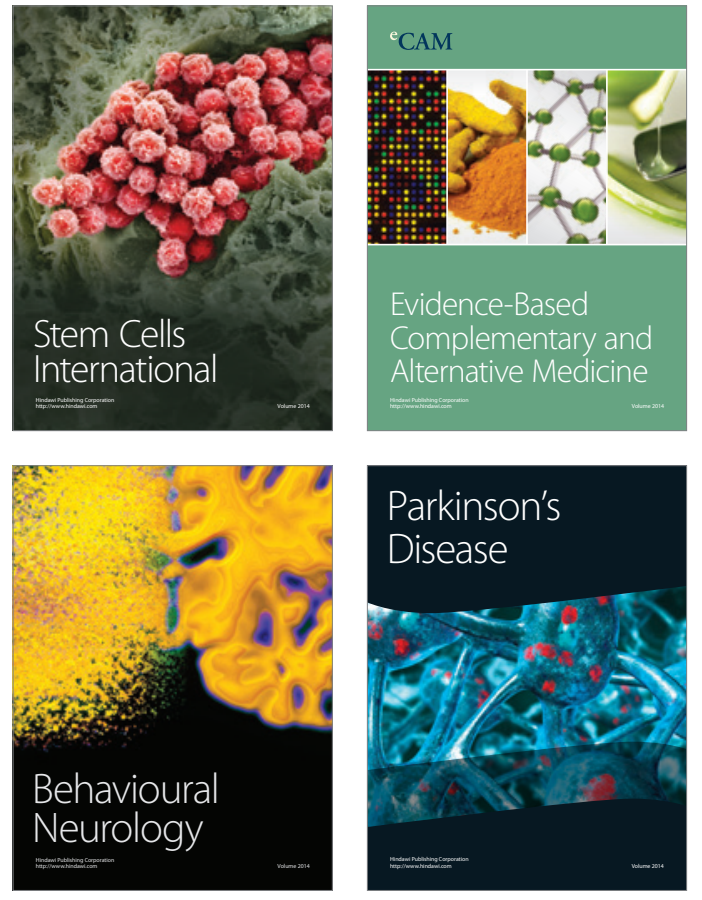
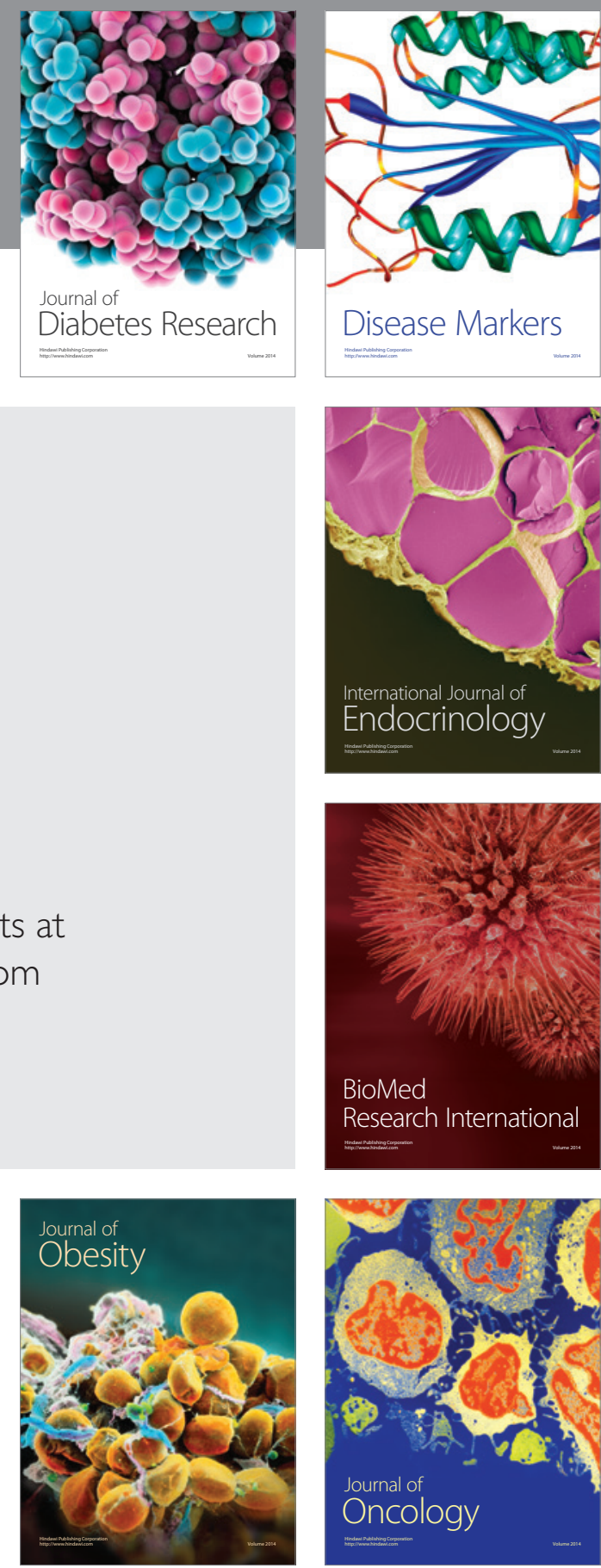

Disease Markers
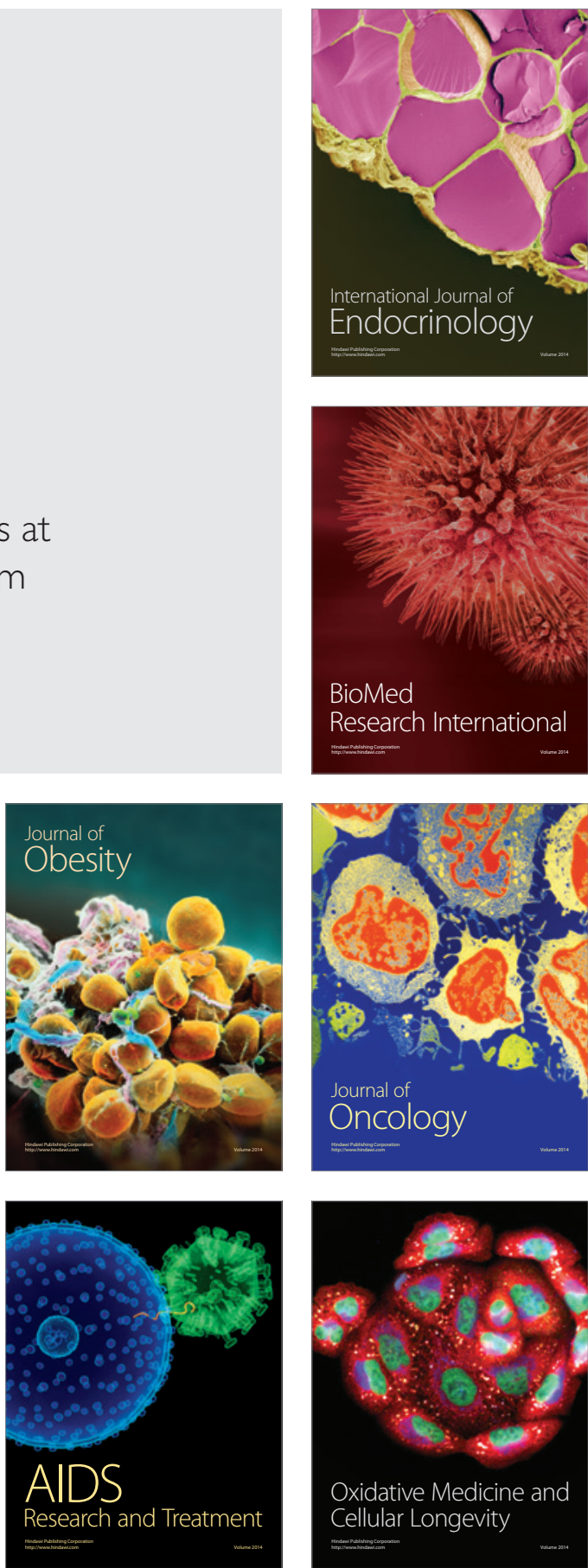